\title{
27. MIOCENE BENTHIC FORAMINIFER OXYGEN AND CARBON ISOTOPES, SITE 709, INDIAN OCEAN1
}

\author{
Fay Woodruff, ${ }^{2}$ Samuel M. Savin, ${ }^{3}$ and Linda $\mathrm{Abel}^{3}$
}

\begin{abstract}
The benthic isotopic record of Miocene Cibicidoides from Site 709 provides a record of conditions in the Indian Ocean at a depth of about 3200 mbsf. As expected, the record qualitatively resembles those of other Deep Sea Drilling Project and Ocean Drilling Program sites. The data are consistent with the scenario for the evolution of thermohaline circulation in the Miocene Indian Ocean proposed by Woodruff and Savin (1989). Further testing of that scenario, however, requires isotopic data for Cibicidoides from other Indian Ocean sites. There is a correlation between $\delta^{13} \mathrm{C}$ values of Cibicidoides and planktonic:benthic (P:B) ratios of Site 709 sediments, implying a causal relationship between the corrosiveness of deep waters and concentration of $\mathrm{CO}_{2}$ derived from oxidation of organic matter.
\end{abstract}

\section{INTRODUCTION}

In this paper, we report the results of oxygen and carbon isotopic analyses of the tests of Miocene benthic foraminifers from Ocean Drilling Program (ODP) Site 709. Site 709 is located near the summit of the Madingley Rise in the western Indian Ocean. Present-day water depth is $3041 \mathrm{~m}$. Back-tracked Miocene depths of the site are between about 2550 and $2900 \mathrm{~m}$, making it the deepest Indian Ocean site for which Miocene benthic isotope data are available.

Woodruff and Savin (1989) presented a global synthesis of Miocene benthic foraminiferal carbon isotopic data and interpreted directions of deep- and intermediate-water flow from variations in $\delta^{13} \mathrm{C}$ values of the tests. They concluded that early Miocene thermohaline circulation was very different from that of today. They proposed that during early Miocene time a plume of warm, saline water from the Tethys entered the northern Indian Ocean in the region of the Red Sea and Persian Gulf. This plume would have sunk to intermediate depths in the Indian Ocean, entraining warm surface water as it sank and forming a water mass they called Tethyan Indian Saline Water (TISW). The TISW would have flowed southward to high southern latitudes where it would have welled up into the circumantarctic surface circulation. There, they proposed, it would have become refrigerated and sunk to the ocean bottom, flowing northward in the Atlantic, Pacific, and Indian oceans analogous to modern Antarctic Bottom Water (AABW). Thus, the proposed Tethyan outflow water and the resulting TISW would have played an early Miocene role similar in some respects to that of North Atlantic Deep Water (NADW) in the modern ocean.

Of all benthic foraminiferal taxa commonly used in isotopic reconstructions of oceanographic conditions, carbon isotopic ratios of the genus Cibicidoides appear to reflect the $\delta^{13} \mathrm{C}$ values of dissolved $\mathrm{HCO}_{3}^{-}$in seawater at the sediment-water interface most closely (Duplessy et al., 1984; Shackleton et al., 1984; Savin and Woodruff, 1990, unpubl. data, and others). Miocene isotopic data are available for only a few sites in the Indian Ocean and most of the available data are for Oridorsalis umbo-

\footnotetext{
${ }^{1}$ Duncan, R. A., Backman, J., Peterson, L. C., et al., 1990. Proc. ODP, Sci. Results, 115: College Station, TX (Ocean Drilling Program).

2 Department of Geological Sciences, University of Southern California, Los Angeles, CA 90089, U.S.A.

3 Department of Geological Sciences, Case Western Reserve University, Cleveland, $\mathrm{OH} 44106$, U.S.A.
}

natus (Vincent et al., 1985). It is critical to the testing of our early Miocene scenario, or any other model of Miocene thermohaline circulation, to enlarge the data base of isotopic compositions of Miocene Cibicidoides from the Indian Ocean. This brief paper represents a small step toward compilation of that data base.

\section{METHODS AND PROCEDURES}

Benthic foraminifers were prepared for isotopic analysis following procedures described by Woodruff and Savin (1989). Preparation of $\mathrm{CO}_{2}$ for isotopic analysis was done with a modified version of the technique of Epstein et al. (1951). These modifications are especially useful in the analysis of small $(<500 \mu \mathrm{g})$ samples of $\mathrm{CaCO}_{3}$.

Samples weighing between a few tens of micrograms and $1 \mathrm{mg}$ were placed in a miniaturized Rittenberg tube (Fig. 1) with $0.5 \mathrm{ml}$ of $100 \%$ phosphoric acid. The tubes were evacuated overnight, and then placed in a thermostated water bath at $25^{\circ} \mathrm{C}$. No more than $24 \mathrm{hr}$ before the $\mathrm{CO}_{2}$ was to be collected, the Rittenberg tube was tipped, which allowed the acid to contact the $\mathrm{CaCO}_{3}$. After a reaction time of $2-24 \mathrm{hr}$ at $25^{\circ} \mathrm{C}$, the tube was plunged into a Dewar flask containing a slush made from a mixture of ethanol and finely ground dry ice. The tube, still in the Dewar, was then attached directly to a mass spectrometer sample inlet system of our own design, and the $\mathrm{CO}_{2}$ was distilled into the variable volume inlet where it was frozen with liquid $\mathrm{N}_{2}$ and analyzed isotopically.

This technique yields cleaner $\mathrm{CO}_{2}$ and isotopic analyses of small samples of higher precision than we could obtain with more conventional multistage cryogenic distillation procedures. We routinely achieved precision of $\delta^{18} \mathrm{O}$ and $\delta^{13} \mathrm{C}$ analyses of better than $0.1 \% 0$ with samples weighing as little as $150 \mu \mathrm{g}$.

\section{RESULTS}

\section{Isotopic Time Series}

Isotopic results, as well as planktonic:benthic $(\mathrm{P}: \mathrm{B})$ ratios of samples from Holes 709A and 709B are tabulated in Table 1. Isotopic data are plotted as a function of depth (meters below sea floor [mbsf]) in Figures $2 a$ and $2 b$. At this time only a few biostratigraphic ages of Miocene Site 709 samples have been determined. We have assigned ages based upon correlations between oxygen and carbon isotopic curves from Holes 709A and $709 \mathrm{~B}$ and those of high-resolution Pacific ${ }^{18} \mathrm{O}$ and ${ }^{13} \mathrm{C}$ records for which there are well-determined nannofossil, radiolarian, diatom, and planktonic foraminifer datums (Woodruff and Savin, 


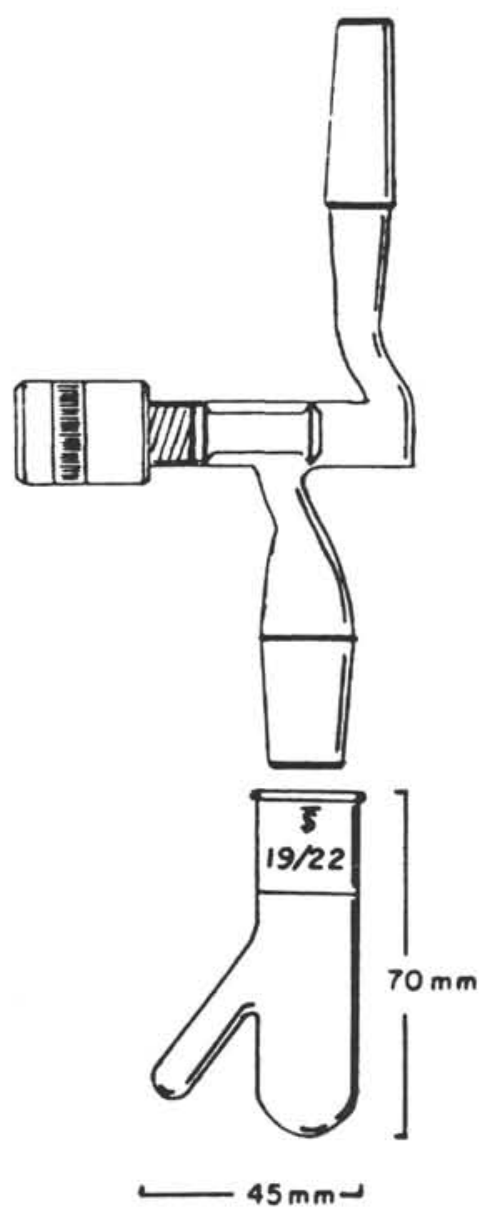

Figure 1. Miniature Rittenberg tube used for reaction of small carbonate samples. The lower portion of the vessel fits easily inside a standard 265-ml Dewar.

unpubl. data). The ages of datums we used are based on those listed in Barron et al., 1985. The early Miocene isotopic patterns are not sufficiently distinct to permit their use for making age assignments. We have a high degree of confidence in the ages of the middle Miocene section between 119 and $150 \mathrm{mbsf}$ from Holes 709A and 709B. We correlated sediments at a depth of 132.5 mbsf from Hole 709A with those from 136 mbsf in Hole 709B as well as sediments from a depth of $151.5 \mathrm{mbsf}$ at Hole $709 \mathrm{~A}$ with those at $150.5 \mathrm{mbsf}$ at Hole $709 \mathrm{~B}$. Thus, the section from Hole 709A fills a gap that we think resulted from sediment missing between Cores 115-709B-15H and -16H. Age determinations based upon the isotopic record and also upon biostratigraphy (Backman, Duncan, et al., 1988; Rio et al., this volume; Johnson, this volume) are indicated in Figure 2; age determinations based on the isotopic record are listed in Table 2. Biostratigraphic age datums from Backman, Duncan, et al. (1988) that appear compatible with the isotopic data are listed in Table 3.

Backman, Duncan, et al. (1988) inferred from whole-core magnetic susceptibility profiles that the early Miocene section from 163.3 to $168.0 \mathrm{mbsf}$ in Hole $709 \mathrm{~B}$ is a repetition of the interval immediately overlying it. The isotopic patterns (Fig. 3) are consistent with, but do not require, this interpretation. Core 115-709B-20H (177.6-187.2 mbsf) was not recovered.

Qualitatively, the major features of the oxygen and carbon isotopic records resemble those of other Miocene sites. These will not be detailed here. Mean $\delta^{18} \mathrm{O}$ and $\delta^{13} \mathrm{C}$ values of Cibicidoides deposited during several Miocene intervals are listed in
Table 4. The mean values, when plotted on the depth vs. latitude transects of Woodruff and Savin (1989), agree for the most part with the patterns and contours suggested by analyses of $\mathrm{Ci}$ bicidoides and adjusted analyses of Oridorsalis from the shallower sites in the Indian Ocean and by analyses of Cibicidoides from a range of depths in the Atlantic and Pacific oceans. That is, the $\delta^{13} \mathrm{C}$ values are only slightly lower than those of shallower benthic foraminifers from the Indian Ocean. However, between 15 and $17 \mathrm{Ma}$, Cibicidoides from Site 709 are depleted in ${ }^{13} \mathrm{C}$ by several tenths of a per mil relative to benthic foraminifers from shallower Indian Ocean sites, and their $\delta^{13} \mathrm{C}$ values are among the lowest found in any ocean during that interval. Interpretation of these unexpectedly low $\delta^{13} \mathrm{C}$ values awaits completion of isotopic analyses of Cibicidoides from other Indian Ocean sites.

\section{Interspecific Isotopic Variations}

The data set provides several opportunities to compare $\delta^{18} \mathrm{O}$ and $\delta^{13} \mathrm{C}$ values of different benthic taxa from within the same sample. The results of these comparisons are listed in Tables 5 and 6. Although there are not a large number of comparisons, the data indicate the possibility that Cibicidoides wuellerstorfi may be depleted in ${ }^{18} \mathrm{O}$ and enriched in ${ }^{13} \mathrm{C}$ by small amounts $(0.0 \%-0.2 \%)$ relative to Cibicidoides kullenbergi and Cibicidoides lamontdohertyi (named by Miller and Katz, 1987). Additional comparisons are necessary to determine whether these isotopic differences are indeed significant. Cibicidoides are depleted in ${ }^{18} \mathrm{O}$ by $0.18-0.49 \%$ (mean $0.38 \%_{0} \pm 0.11 \% 0$ ) relative to Oridorsalis umbonatus from the same samples. Cibicidoides are enriched in ${ }^{13} \mathrm{C}$ relative to Oridorsalis by amounts ranging from $-0.56 \%_{0}$ to $-1.22 \%_{0}$ (mean $0.96 \%_{0} \pm 0.26 \%$ ). The mean differences are similar to the adjustments used by Woodruff and Savin (1989) to adjust Oridorsalis $\delta$ values to those expected for Cibicidoides from the same samples $(0.60$ for oxygen and -1.10 for carbon. The standard deviation about the mean difference for carbon is large, however.

\section{Relation between $\delta^{13} \mathrm{C}$ Values and Planktonic:Benthic Ratios}

Planktonic:benthic ratios depend in part upon productivity and the rate of sedimentation of planktonic foraminifers, and in part on the degree of preservation of carbonate on the seafloor. Because planktonic tests are, in general, more readily dissolved than benthic tests, the $\mathrm{P}: \mathrm{B}$ ratio decreases as foraminifers are dissolved at the sediment-water interface. At Site 709 there is a striking correlation between P:B ratios and the $\delta^{13} \mathrm{C}$ values of Cibicidoides. This may be seen in the time series graph of Figure 4. (The logarithm of the P:B ratio is plotted because of the extremely wide range of variability of the ratio.). Although the $\mathrm{P}: \mathrm{B}$ record is especially noisy, it is clear that higher $\delta^{13} \mathrm{C}$ values correspond to higher $\mathrm{P}: \mathrm{B}$ ratios. A linear regression indicates a correlation between $\delta^{13} \mathrm{C}$ and a 5-point running average of log P:B (Fig. 5) that is significant at the $99 \%$ level. This correlation strongly suggests that P:B ratios at Site 709 reflect conditions at the sediment-water interface to a significant extent.

The $\delta^{13} \mathrm{C}$ values of deep and intermediate waters are affected by a complex of factors related to the input of organic and inorganic carbon to the oceans, the oxidation of organic carbon in seawater, and removal of carbon into sediments. In a global synoptic view, $\delta^{13} \mathrm{C}$ values of dissolved $\mathrm{HCO}_{3}^{-}$in deep and intermediate waters are negatively correlated with the amount of dissolved respiratory $\mathrm{CO}_{2}$ in the water. The dissolution of respiratory $\mathrm{CO}_{2}$ also enhances the solubility of $\mathrm{CaCO}_{3}$ in the water.

Temporal variations in the $\delta^{13} \mathrm{C}$ value of dissolved $\mathrm{HCO}_{3}^{-}$at a single site cannot be interpreted so simply. However, the correlation between $\delta^{13} \mathrm{C}$ and $\mathrm{P}: \mathrm{B}$ ratios at Site 709 , and the general similarity between the $\delta^{13} \mathrm{C}$ record of Site 709 and those of other Atlantic, Pacific, and Indian ocean sites imply that intervals in 
Table 1. Oxygen and carbon isotope ratios of Miocene benthic foraminifers from Site 709A and 709B.

\begin{tabular}{|c|c|c|c|c|c|}
\hline $\begin{array}{l}\text { Core, section, } \\
\text { interval }(\mathrm{cm})\end{array}$ & $\begin{array}{l}\text { Depth } \\
\text { (mbsf) }\end{array}$ & Species & $\delta^{18} \mathrm{O}$ & $\delta^{13} \mathrm{C}$ & $\begin{array}{l}\mathrm{P}: \mathrm{B} \\
\text { ratio }\end{array}$ \\
\hline \multicolumn{6}{|l|}{$115-709 \mathrm{~A}-$} \\
\hline $14 \mathrm{H}-5,2-5$ & 131.8 & Cibicidoides kullenbergi & 1.59 & 0.75 & 371 \\
\hline $14 \mathrm{H}-5,50-53$ & 132.3 & Cibicidoides kullenbergi & 1.69 & 1.01 & 796 \\
\hline $14 \mathrm{H}-5,94-97$ & 132.7 & Cibicidoides kullenbergi & 1.44 & 0.59 & 351 \\
\hline $14 \mathrm{H}-6,1-4$ & 133.31 & Cibicidoides kullenbergi & 1.76 & 0.86 & 365 \\
\hline $14 \mathrm{H}-6,50-53$ & 133.80 & Cibicidoides kullenbergi & 1.54 & 0.67 & 230 \\
\hline $14 \mathrm{H}-6,100-103$ & 134.30 & Cibicidoides kullenbergi & 1.78 & 0.80 & 430 \\
\hline $14 \mathrm{H}-7,3-6$ & 134.83 & Cibicidoides kullenbergi & 1.76 & 0.73 & 484 \\
\hline $14 \mathrm{H}-7,47-50$ & 135.3 & $\begin{array}{l}\text { Cibicidoides kullenbergi } \\
\quad+\text { C. lamontdohertyi }\end{array}$ & 1.53 & 0.31 & 103 \\
\hline $15 \mathrm{H}-1,5-8$ & 135.55 & Cibicidoides kullenbergi & 1.76 & 0.88 & 180 \\
\hline $15 \mathrm{H}-1,50-53$ & 136.00 & Cibicidoides kullenbergi & 1.86 & 0.85 & 145 \\
\hline $15 \mathrm{H}-1,101-104$ & 136.51 & Cibicidoides kullenbergi & 1.75 & 0.77 & 208 \\
\hline $15 \mathrm{H}-2,2-5$ & 137.02 & Cibicidoides kullenbergi & 1.70 & 0.56 & 150 \\
\hline $15 \mathrm{H}-2,50-53$ & 137.501 & Cibicidoides lamontdohertyi & 1.74 & 0.81 & 52 \\
\hline $15 \mathrm{H}-2,94-97$ & 137.94 & $\begin{array}{l}\text { Cibicidoides kullenbergi } \\
\quad+C \text {. lamontdohertyi }\end{array}$ & 1.73 & 0.78 & 29 \\
\hline $15 \mathrm{H}-3,2-5$ & 138.52 & Cibicidoides lamontdohertyi & 1.99 & 0.64 & 74 \\
\hline $15 \mathrm{H}-3,2-5$ & 138.52 & Cibicidoides kullenbergi & 1.79 & 0.65 & 74 \\
\hline $15 \mathrm{H}-3,50-53$ & 139.00 & Cibicidoides kullenbergi & 1.99 & 0.86 & 95 \\
\hline $15 \mathrm{H}-3,50-53$ & 139.00 & Cibicidoides lamontdohertyi & 2.12 & 0.70 & 95 \\
\hline $15 \mathrm{H}-3,100-103$ & 139.50 & Cibicidoides kullenbergi & 1.67 & 0.87 & 102 \\
\hline $15 \mathrm{H}-4,2-5$ & 140.02 & Cibicidoides kullenbergi & 1.83 & 0.90 & 201 \\
\hline $15 \mathrm{H}-4,50-53$ & 140.50 & Cibicidoides kullenbergi & 1.84 & 0.72 & 154 \\
\hline $15 \mathrm{H}-4,94-97$ & 140.94 & Cibicidoides kullenbergi & 1.89 & 1.53 & 167 \\
\hline $15 \mathrm{H}-5,2-5$ & 141.52 & Cibicidoides kullenbergi & 1.59 & 1.64 & 106 \\
\hline $15 \mathrm{H}-5,50-53$ & 142.00 & $\begin{array}{l}\text { Cibicidoides kullenbergi } \\
\quad+C \text {. lamontdohertyi }\end{array}$ & 1.72 & 1.84 & 137 \\
\hline $15 \mathrm{H}-5,94-97$ & 142.54 & Cibicidoides kullenbergi & 1.75 & 1.76 & 390 \\
\hline $15 \mathrm{H}-6,2-5$ & 143.02 & $\begin{array}{l}\text { Cibicidoides kullenbergi } \\
\quad+\text { C. } \mathrm{sp} . \mathrm{S}+\text { C. trinitatensis }\end{array}$ & 1.44 & 1.80 & 545 \\
\hline $15 \mathrm{H}-6,50-53$ & 143.50 & Cibicidoides kullenbergi & 1.76 & 1.63 & 566 \\
\hline $15 \mathrm{H}-6,100-103$ & 144.00 & $\begin{array}{l}\text { Cibicidoides kullenbergi } \\
\quad+C \text {. lamontdohertyi }+ \text { C. trinitatensis }\end{array}$ & 1.34 & 1.59 & 404 \\
\hline $15 \mathrm{H}-7,2-5$ & 144.52 & $\begin{array}{l}\text { Cibicidoides kullenbergi } \\
\quad+\text { C. lamontdohertyi }\end{array}$ & 1.54 & 1.58 & 461 \\
\hline $15 \mathrm{H}-7,50-52$ & 145.00 & $\begin{array}{l}\text { Cibicidoides kullenbergi } \\
\quad+C . \mathrm{sp} . \mathrm{S}\end{array}$ & 1.50 & 1.58 & 493 \\
\hline $16 \mathrm{H}-1,2-5$ & 145.22 & $\begin{array}{l}\text { Cibicidoides kullenbergi } \\
\quad+C . \mathrm{sp} . \mathrm{S}\end{array}$ & 1.65 & 1.54 & 181 \\
\hline $16 \mathrm{H}-1,50-53$ & 145.70 & $\begin{array}{l}\text { Cibicidoides kullenbergi } \\
\quad+C . \mathrm{sp} . \mathrm{S}\end{array}$ & 1.42 & 1.25 & 696 \\
\hline $16 \mathrm{H}-1,94-97$ & 146.14 & $\begin{array}{l}\text { Cibicidoides kullenbergi } \\
\quad+C . \mathrm{sp} . \mathrm{S}\end{array}$ & 1.46 & 1.20 & 294 \\
\hline $16 \mathrm{H}-2,2-5$ & 146.72 & $\begin{array}{l}\text { Cibicidoides kullenbergi } \\
\quad+\text { C. } \mathrm{sp} . \mathrm{S}+\text { C. lamontdohertyi }\end{array}$ & 1.66 & 1.51 & 465 \\
\hline $16 \mathrm{H}-2,50-52$ & 147.20 & Cibicidoides kullenbergi & 1.19 & 0.97 & 416 \\
\hline $16 \mathrm{H}-2,94-97$ & 147.64 & $\begin{array}{l}\text { Cibicidoides wuellerstorfi } \\
+ \text { C. lamontdohertyi }\end{array}$ & 1.37 & 0.99 & 199 \\
\hline $16 \mathrm{H}-3,2-5$ & 148.22 & $\begin{array}{l}\text { Cibicidoides kullenbergi } \\
\quad+\text { C. lamontdohertyi }\end{array}$ & 1.87 & 1.08 & 241 \\
\hline $16 \mathrm{H}-3,50-53$ & 148.70 & Cibicidoides kullenbergi & 1.71 & 0.97 & 557 \\
\hline $16 \mathrm{H}-3,101-104$ & 149.21 & $\begin{array}{l}\text { Cibicidoides kullenbergi } \\
\quad+\text { C. lamontdohertyi }\end{array}$ & 1.73 & 1.03 & 131 \\
\hline $16 \mathrm{H}-4,2-5$ & 149.72 & $\begin{array}{l}\text { Cibicidoides kullenbergi } \\
\quad+\text { C. lamontdohertyi }\end{array}$ & 1.98 & 1.10 & 519 \\
\hline $16 \mathrm{H}-4,50-53$ & 150.20 & Cibicidoides kullenbergi & 1.52 & 0.81 & 347 \\
\hline $16 \mathrm{H}-4,102-105$ & 150.72 & $\begin{array}{l}\text { Cibicidoides kullenbergi } \\
\quad+C \text {. lamontdohertyi }\end{array}$ & 1.86 & 0.80 & 423 \\
\hline $16 \mathrm{H}-5,2-5$ & 151.22 & Planulina renzi & 1.50 & 0.85 & 249 \\
\hline $16 \mathrm{H}-5,2-5$ & 151.22 & Cibicidoides kullenbergi & 1.70 & 0.76 & 249 \\
\hline $16 \mathrm{H}-5,50-53$ & 151.70 & Cibicidoides kullenbergi & 1.64 & 0.76 & 189 \\
\hline $16 \mathrm{H}-5,94-97$ & 152.20 & $\begin{array}{l}\text { Cibicidoides kullenbergi } \\
\quad+\text { C. lamontdohertyi }\end{array}$ & 1.86 & 0.85 & 273 \\
\hline $16 \mathrm{H}-6,2-5$ & 152.70 & Cibicidoides kullenbergi & 1.60 & 0.66 & 278 \\
\hline $16 \mathrm{H}-6,53-56$ & 153.23 & Cibicidoides kullenbergi & 1.69 & 0.79 & 287 \\
\hline $16 \mathrm{H}-6,100-103$ & 153.73 & Cibicidoides kullenbergi & 1.53 & 0.70 & 227 \\
\hline $16 \mathrm{H}-7,2-5$ & 154.22 & Cibicidoides kullenbergi & 1.80 & 0.69 & 157 \\
\hline $16 \mathrm{H}-7,50-53$ & 154.70 & Cibicidoides kullenbergi & 1.51 & 0.45 & 111 \\
\hline $17 \mathrm{H}-1,50-53$ & 155.3 & $\begin{array}{l}\text { Cibicidoides kullenbergi } \\
+ \text { Planulina renzi }\end{array}$ & 1.87 & 1.02 & \\
\hline $17 \mathrm{H}-1,100-103$ & 155.9 & $\begin{array}{l}\text { Cibicidoides kullenbergi } \\
+ \text { Planulina renzi }\end{array}$ & 1.87 & 1.11 & \\
\hline $17 \mathrm{H}-2,4-7$ & 156.5 & $\begin{array}{l}\text { Cibicidoides kullenbergi } \\
+ \text { Planulina renzi }\end{array}$ & 1.64 & 0.91 & \\
\hline
\end{tabular}


F. WOODRUFF, S. M. SAVIN, L. ABEL

Table 1 (continued).

\begin{tabular}{|c|c|c|c|c|c|}
\hline $\begin{array}{l}\text { Core, section, } \\
\text { interval }(\mathrm{cm})\end{array}$ & $\begin{array}{l}\text { Depth } \\
\text { (mbsf) }\end{array}$ & Species & $\delta^{18} \mathrm{O}$ & $\delta^{13} \mathrm{C}$ & $\begin{array}{l}\mathrm{P}: \mathrm{B} \\
\text { ratio }\end{array}$ \\
\hline \multicolumn{6}{|l|}{ 115-709B- } \\
\hline $7 \mathrm{H}-2,75-80$ & 54.2 & Cibicidoides wuellerstorfi & 2.63 & 0.03 & 167 \\
\hline $7 \mathrm{H}-4,70-75$ & 57.2 & $\begin{array}{l}\text { C. whellerstorfi } \\
\quad+C . \text { kullenbergi }+ \text { Planulina bradyi }\end{array}$ & 2.84 & 0.37 & 244 \\
\hline $7 \mathrm{H}-5,125-130$ & 59.2 & $\begin{array}{l}\text { C. wuellerstorfi } \\
\quad+\text { Planulina bradyi }\end{array}$ & 2.62 & 0.24 & 357 \\
\hline $8 \mathrm{H}-2,25-30$ & 61.9 & $\begin{array}{l}\text { Cibicidoides kullenbergi } \\
\quad+C \text { wuellerstorfi }\end{array}$ & 2.73 & 0.16 & 57 \\
\hline $8 \mathrm{H}-4,70-75$ & 66.9 & Cibicidoides wuellerstorfi & 2.68 & -0.12 & 274 \\
\hline $8 \mathrm{H}-5,125-130$ & 68.9 & Cibicidoides wuellerstorfi & 2.74 & 0.22 & 34 \\
\hline $9 \mathrm{H}-2,25-30$ & 71.9 & Cibicidoides wuellerstorfi & 2.71 & 0.27 & 25 \\
\hline $9 \mathrm{H}-4,70-75$ & 76.5 & $\begin{array}{l}\text { Cibicidoides rugosa } \\
\quad+\text { C. kullenbergi }\end{array}$ & 2.89 & 0.29 & 262 \\
\hline $9 \mathrm{H}-5,125-130$ & 78.3 & Cibicidoides wuellerstorfi & 2.81 & 0.05 & 87 \\
\hline $10 \mathrm{H}-2,25-30$ & 82.7 & $\begin{array}{l}\text { Cibicidoides wuellerstorfi } \\
\quad+\text { C. rugosa }\end{array}$ & 2.66 & 0.04 & 57 \\
\hline $10 \mathrm{H}-4,70-75$ & 86.2 & Cibicidoides wuellerstorfi & 2.61 & 0.71 & 231 \\
\hline $10 \mathrm{H}-5,125-130$ & 88.2 & Cibicidoides kullenbergi & 2.66 & 0.54 & 175 \\
\hline $11 \mathrm{H}-2,25-30$ & 92.3 & Oridorsalis umbonatus & 2.97 & -1.10 & 289 \\
\hline $11 \mathrm{H}-2,25-30$ & 92.3 & Cibicidoides wuellerstorfi & 2.53 & 0.12 & 289 \\
\hline $11 \mathrm{H}-2,25-30$ & 92.3 & Cibicidoides sp. B & 2.57 & 0.15 & 289 \\
\hline $11 \mathrm{H}-2,25-30$ & 92.3 & Planulina bradyi & 2.98 & 0.78 & 289 \\
\hline $11 \mathrm{H}-4,25-30$ & 95.3 & $\begin{array}{l}\text { Cibicidoides kullenbergi } \\
\quad+\text { C. wuellerstorfi }+ \text { C. sp. B }\end{array}$ & 2.67 & 1.10 & 250 \\
\hline $11 \mathrm{H}-4,70-75$ & 95.8 & Cibicidoides wuellerstorfi & 2.53 & 1.11 & 221 \\
\hline $11 \mathrm{H}-6,25-30$ & 98.3 & Oridorsalis umbonatus & 3.08 & 0.42 & 270 \\
\hline $11 \mathrm{H}-6,25-30$ & 98.3 & Cibicidoides wuellerstorfi & 2.59 & 0.76 & 270 \\
\hline $11 \mathrm{H}-6,25-30$ & 98.3 & Cibicidoides kullenbergi & 2.67 & 0.63 & 270 \\
\hline $12 \mathrm{H}-2,25-30$ & 102.0 & $\begin{array}{l}\text { C. wuellerstorfi } \\
+ \text { Planulina bradyi }\end{array}$ & 2.51 & 0.94 & 301 \\
\hline $12 \mathrm{H}-3,70-75$ & 104.0 & Cibicidoides wuellerstorfi & 2.72 & 0.60 & 670 \\
\hline $12 \mathrm{H}-4,25-30$ & 105.0 & Cibicidoides wuellerstorfi & 2.55 & 0.70 & 329 \\
\hline $12 \mathrm{H}-7,25-30$ & 109.2 & Cibicidoides kullenbergi & 2.78 & 0.72 & 202 \\
\hline $13 \mathrm{H}-1,75-80$ & 110.6 & Cibicidoides wuellerstorfi & 2.68 & 0.54 & 74 \\
\hline $13 \mathrm{H}-1,125-130$ & 111.1 & Cibicidoides wuellerstorfi & 2.49 & 0.59 & 135 \\
\hline $13 \mathrm{H}-2,25-30$ & & & & & 38 \\
\hline $13 \mathrm{H}-2,75-80$ & 112.15 & Cibicidoides wuellerstorfi & 2.52 & 0.70 & 37 \\
\hline $13 \mathrm{H}-2,125-130$ & 112.65 & Cibicidoides wuellerstorfi & 2.39 & 0.56 & 43 \\
\hline $13 \mathrm{H}-3,25-30$ & 113.15 & Cibicidoides kullenbergi & 2.58 & 0.45 & 80 \\
\hline $13 \mathrm{H}-3,70-75$ & 113.60 & Cibicidoides wuellerstorfi & 2.52 & 0.90 & 490 \\
\hline $13 \mathrm{H}-3,70-75$ & 113.60 & Cibicidoides kullenbergi & 2.63 & 0.86 & 490 \\
\hline $13 \mathrm{H}-3,75-80$ & 113.65 & Cibicidoides kullenbergi & 2.48 & 0.78 & 69 \\
\hline $13 \mathrm{H}-3,75-80$ & 113.65 & Cibicidoides wuellerstorfi & 2.56 & 0.93 & 69 \\
\hline $13 \mathrm{H}-3,125-130$ & 114.1 & Cibicidoides wuellerstorfi & 2.32 & 0.80 & 41 \\
\hline $13 \mathrm{H}-4,25-30$ & 114.7 & Cibicidoides wuellerstorfi & 2.53 & 0.71 & 113 \\
\hline $13 \mathrm{H}-4,75-80$ & 115.1 & Cibicidoides wuellerstorfi & 2.46 & 0.77 & 33 \\
\hline $13 \mathrm{H}-4,125-130$ & 115.6 & Cibicidoides kullenbergi & 2.56 & 0.62 & 58 \\
\hline $13 \mathrm{H}-4,125-130$ & 115.6 & Cibicidoides wuellerstorfi & 2.33 & 0.86 & 58 \\
\hline $13 \mathrm{H}-5,25-30$ & 116.1 & Cibicidoides lamontdohertyi & 2.62 & 0.60 & 140 \\
\hline $13 \mathrm{H}-5,25-30$ & 116.1 & Cibicidoides wuellerstorfi & 2.37 & 0.93 & 140 \\
\hline $13 \mathrm{H}-5,75-80$ & 116.6 & Cibicidoides lamontdohertyi & 2.57 & 0.67 & 148 \\
\hline $13 \mathrm{H}-5,75-80$ & 116.6 & Cibicidoides kullenbergi & 2.65 & 0.78 & 148 \\
\hline $13 \mathrm{H}-5,75-80$ & 116.6 & Cibicidoides wuellerstorfi & 2.33 & 0.72 & 148 \\
\hline $13 \mathrm{H}-5,125-130$ & 117.1 & Cibicidoides wuellerstorfi & 2.38 & 1.14 & 75 \\
\hline $13 \mathrm{H}-6,25-30$ & 117.6 & Cibicidoides wuellerstorfi & 2.59 & 1.05 & 353 \\
\hline $13 \mathrm{H}-6,75-80$ & 118.1 & Cibicidoides wuellerstorfi & 2.34 & 1.21 & 272 \\
\hline $13 \mathrm{H}-6,125-130$ & 118.6 & Cibicidoides wuellerstorfi & 2.38 & 0.97 & 266 \\
\hline $13 \mathrm{H}-7,25-30$ & 119.1 & Cibicidoides wuellerstorfi & 2.37 & 0.94 & 303 \\
\hline $14 \mathrm{H}-1,75-80$ & 120.3 & $\begin{array}{l}\text { Cibicidoides wuellerstorfi } \\
\quad+\text { C. } \mathrm{sp} . \mathrm{C}+\text { C. kullenbergi }\end{array}$ & 2.33 & 1.56 & 601 \\
\hline $14 \mathrm{H}-1,125-130$ & 120.8 & Cibicidoides wuellerstorfi & 2.50 & 0.99 & 114 \\
\hline $14 \mathrm{H}-1,125-130$ & 120.8 & Cibicidoides lamontdohertyi & 2.57 & 0.72 & 114 \\
\hline $14 \mathrm{H}-2,25-30$ & 121.3 & $\begin{array}{l}\text { Cibicidoides wuellerstorfi } \\
\quad+\text { C. kullenbergi }\end{array}$ & 2.29 & 0.67 & 386 \\
\hline $14 \mathrm{H}-2,75-80$ & 121.8 & $\begin{array}{l}\text { Cibicidoides kullenbergi } \\
\quad+C . \text { wuellerstorfi }\end{array}$ & 2.56 & 0.92 & 302 \\
\hline $14 \mathrm{H}-2,125-130$ & 122.3 & $\begin{array}{l}\text { Cibicidoides kullenbergi } \\
\quad+C \text {. lamontdohertyi }\end{array}$ & 2.60 & 0.82 & 309 \\
\hline $14 \mathrm{H}-3,25-30$ & 122.8 & Cibicidoides lamontdohertyi & 2.66 & 0.81 & 372 \\
\hline $14 \mathrm{H}-3,70-75$ & 123.2 & Oridorsalis umbonatus & 2.65 & -0.85 & 210 \\
\hline $14 \mathrm{H}-3,75-80$ & 123.3 & $\begin{array}{l}\text { Cibicidoides kullenbergi } \\
\quad+\text { C. lamontdohertyi }\end{array}$ & 2.36 & 0.78 & 127 \\
\hline $14 \mathrm{H}-3,125-130$ & 123.8 & Cibicidoides kullenbergi & 2.49 & 0.99 & 142 \\
\hline $14 \mathrm{H}-4,25-30$ & 124.3 & $\begin{array}{l}\text { Cibicidoides kullenbergi } \\
\quad+\text { C. wuellerstorfi }\end{array}$ & 2.51 & 0.95 & 322 \\
\hline $14 \mathrm{H}-4,75-80$ & 124.8 & $\begin{array}{l}\text { Cibicidoides kullenbergi } \\
\quad+\text { C. wuellerstorfi }+ \text { C. lamontdohertyi }\end{array}$ & 2.49 & 0.99 & 481 \\
\hline $14 \mathrm{H}-4,125-130$ & 125.3 & Cibicidoides kullenbergi & 2.28 & 1.05 & 198 \\
\hline
\end{tabular}


Table 1 (continued).

\begin{tabular}{|c|c|c|c|c|c|}
\hline $\begin{array}{l}\text { Core, section, } \\
\text { interval }(\mathrm{cm})\end{array}$ & $\begin{array}{l}\text { Depth } \\
\text { (mbsf) }\end{array}$ & Species & $\delta^{18} \mathrm{O}$ & $\delta^{13} \mathrm{C}$ & $\begin{array}{l}\mathrm{P}: \mathrm{B} \\
\text { ratio }\end{array}$ \\
\hline \multicolumn{6}{|l|}{ 115-709B- (Cont.) } \\
\hline $14 \mathrm{H}-5,25-30$ & 125.7 & $\begin{array}{l}\text { Cibicidoides kullenbergi } \\
+ \text { C. wuellerstorfi }\end{array}$ & 2.12 & 1.65 & 591 \\
\hline $14 \mathrm{H}-5,125-130$ & 126.8 & $\begin{array}{l}\text { Cibicidoides kullenbergi } \\
\quad+C . \text { wuellerstorfi }\end{array}$ & 2.22 & 1.79 & 1294 \\
\hline $14 \mathrm{H}-6,25-30$ & 127.2 & $\begin{array}{l}\text { Cibicidoides kullenbergi } \\
\quad+C \text {. wuellerstorfi }+C \text {. lamontdohertyi }\end{array}$ & 2.38 & 1.01 & 398 \\
\hline $14 \mathrm{H}-6,75-80$ & 127.8 & $\begin{array}{l}\text { Cibicidoides kullenbergi } \\
\quad+\text { C. wuellerstorfi }+ \text { C. lamontdohertyi }\end{array}$ & 2.25 & 1.19 & 390 \\
\hline $14 \mathrm{H}-6,125-130$ & 128.3 & $\begin{array}{l}\text { Cibicidoides wuellerstorfi } \\
\quad+C . \text { kullenbergi }\end{array}$ & 2.11 & 1.25 & 423 \\
\hline $14 \mathrm{H}-7,25-30$ & 128.7 & $\begin{array}{l}\text { Cibicidoides wuellerstorfi } \\
\quad+C . \text { kullenbergi }\end{array}$ & 2.13 & 1.80 & 579 \\
\hline $15 \mathrm{H}-1,75-80$ & 130.0 & Cibicidoides kullenbergi $+C$. sp. C & 1.66 & 1.38 & 200 \\
\hline $15 \mathrm{H}-1,125-130$ & 130.5 & Cibicidoides kullenbergi & 1.70 & 1.33 & \\
\hline $15 \mathrm{H}-2,25-30$ & 130.9 & $\begin{array}{l}\text { Cibicidoides kullenbergi } \\
\quad+\text { C. lamontdohertyi }+ \text { Planulina bradyi }\end{array}$ & 1.69 & 1.19 & 442 \\
\hline $15 \mathrm{H}-2,70-75$ & 131.5 & Cibicidoides kullenbergi & 1.50 & 1.56 & 75 \\
\hline $15 \mathrm{H}-2,125-130$ & 132.0 & Cibicidoides kullenbergi & 1.84 & 1.46 & \\
\hline $15 \mathrm{H}-3,25-30$ & 132.4 & Cibicidoides kullenbergi & 1.19 & 1.41 & \\
\hline $15 \mathrm{H}-3,70-75$ & 132.9 & Cibicidoides kullenbergi & 1.32 & 1.08 & 152 \\
\hline $15 \mathrm{H}-3,75-80$ & 133.0 & Cibicidoides kullenbergi & 1.56 & 1.35 & \\
\hline $15 \mathrm{H}-3,125-130$ & 133.5 & Planulina bradyi & 2.00 & 1.11 & 70 \\
\hline $15 \mathrm{H}-3,125-130$ & 133.5 & Cibicidoides kullenbergi & 1.82 & 1.47 & 70 \\
\hline $15 \mathrm{H}-4,25-30$ & 133.9 & Oridorsalis umbonatus & 2.29 & 0.94 & 262 \\
\hline $15 \mathrm{H}-4,25-30$ & 133.9 & Cibicidoides kullenbergi & 1.80 & 1.53 & 262 \\
\hline $15 \mathrm{H}-4,75-80$ & 134.5 & Cibicidoides lamontdohertyi & 2.15 & 1.12 & 65 \\
\hline $15 \mathrm{H}-4,125-130$ & 135.0 & Cibicidoides kullenbergi & 1.90 & 1.08 & \\
\hline $15 \mathrm{H}-5,23-30$ & 135.4 & Oridorsalis umbonatus & 2.10 & 0.27 & 233 \\
\hline $15 \mathrm{H}-5,25-30$ & 135.4 & Cibicidoides kullenbergi & 1.75 & 1.03 & 433 \\
\hline $15 \mathrm{H}-5,75-80$ & 136.0 & $\begin{array}{l}\text { Cibicidoides kullenbergi } \\
\quad+C \text {. lamontdohertyi }\end{array}$ & 1.47 & 0.57 & 70 \\
\hline $15 \mathrm{H}-5,125-130$ & 136.5 & Cibicidoides kullenbergi & 1.52 & 0.64 & \\
\hline $15 \mathrm{H}-6,25-30$ & 136.9 & $\begin{array}{l}\text { Cibicidoides kullenbergi } \\
\quad+\text { C. lamontdohertyi + Planulina renzi }\end{array}$ & 1.80 & 0.70 & 247 \\
\hline $15 \mathrm{H}-6,70-75$ & 137.5 & Cibicidoides kullenbergi & 1.82 & 0.60 & 30 \\
\hline $15 \mathrm{H}-6,125-130$ & 138.0 & $\begin{array}{l}\text { Cibicidoides kullenbergi } \\
\quad+\text { C. lamontdohertyi }\end{array}$ & 1.75 & 0.79 & \\
\hline $15 \mathrm{H}-7,25-30$ & 138.4 & Cibicidoides kullenbergi & 1.76 & 0.88 & 256 \\
\hline $16 \mathrm{H}-1,25-30$ & 139.2 & Oridorsalis umbonatus & 1.76 & 0.05 & 498 \\
\hline $16 \mathrm{H}-1,25-30$ & 139.2 & $\begin{array}{l}\text { Cibicidoides kullenbergi } \\
\quad+\text { C. lamontdohertyi }\end{array}$ & 1.48 & 1.06 & 498 \\
\hline $16 \mathrm{H}-1,75-80$ & 139.7 & Cibicidoides kullenbergi & 1.49 & 1.34 & 146 \\
\hline $16 \mathrm{H}-1,125-130$ & 140.2 & Cibicidoides kullenbergi $>250 \mu \mathrm{m}$ & 1.77 & 1.38 & 437 \\
\hline $16 \mathrm{H}-1,125-130$ & 140.2 & Cibicidoides kullenbergi $<250 \mu \mathrm{m}$ & 1.80 & 1.49 & 437 \\
\hline $16 \mathrm{H}-2,25-30$ & 140.6 & Cibicidoides kullenbergi & 1.26 & 1.35 & 91 \\
\hline $16 \mathrm{H}-2,25-30$ & 140.6 & Planulina renzi & 1.49 & 1.66 & 91 \\
\hline $16 \mathrm{H}-2,70-75$ & 141.1 & Cibicidoides kullenbergi & 1.61 & 1.71 & 482 \\
\hline $16 \mathrm{H}-2,125-130$ & 141.7 & Cibicidoides kullenbergi & 1.78 & 1.93 & 435 \\
\hline $16 \mathrm{H}-3,25-30$ & 142.1 & Cibicidoides kullenbergi & 1.60 & 1.70 & 496 \\
\hline $17 \mathrm{H}-2,70-75$ & 149.3 & Oridorsalis umbonatus & 2.27 & 0.17 & 297 \\
\hline $18 \mathrm{H}-1,25-30$ & 158.5 & $\begin{array}{l}\text { Cibicidoides kullenbergi } \\
\quad+\text { Planulina renzi }\end{array}$ & 1.76 & 0.67 & 195 \\
\hline $18 \mathrm{H}-1,75-80$ & 159.0 & Cibicidoides kullenbergi & 1.68 & 0.83 & 230 \\
\hline $18 \mathrm{H}-1,125-130$ & 159.5 & Cibicidoides kullenbergi & 1.37 & 0.63 & 113 \\
\hline $18 \mathrm{H}-2,25-30$ & 160.0 & Cibicidoides kullenbergi & 1.68 & 0.45 & 111 \\
\hline $18 \mathrm{H}-2,70-75$ & 160.5 & Cibicidoides kullenbergi & 1.65 & 0.62 & 196 \\
\hline $18 \mathrm{H}-2,125-130$ & 161.0 & $\begin{array}{l}\text { Cibicidoides kullenbergi } \\
\quad+\text { C. lamontdohertyi }\end{array}$ & 1.78 & 0.71 & 320 \\
\hline $18 \mathrm{H}-3,25-30$ & 161.5 & $\begin{array}{l}\text { Cibicidoides kullenbergi } \\
\quad+\text { C. sp. S }\end{array}$ & 1.78 & 0.73 & 137 \\
\hline $18 \mathrm{H}-3,75-80$ & 162.0 & $\begin{array}{l}\text { Cibicidoides kullenbergi } \\
\quad+\text { C. lamontdohertyi }\end{array}$ & 1.86 & 0.75 & 263 \\
\hline $18 \mathrm{H}-3,125-130$ & 162.5 & $\begin{array}{l}\text { Cibicidoides kullenbergi } \\
\quad+\text { C. lamontdohertyi }\end{array}$ & 1.79 & 0.84 & 597 \\
\hline $18 \mathrm{H}-4,25-30$ & 163.0 & Cibicidoides kullenbergi & 1.47 & 0.72 & 183 \\
\hline $18 \mathrm{H}-5,25-30$ & 164.5 & Cibicidoides kullenbergi & 1.53 & 0.49 & 63 \\
\hline $18 \mathrm{H}-6,25-30$ & 166.0 & Cibicidoides kullenbergi & 1.70 & 0.77 & 117 \\
\hline $18 \mathrm{H}-7,25-30$ & 167.5 & Cibicidoides kullenbergi & 1.75 & 0.75 & 132 \\
\hline $19 \mathrm{H}-1,25-30$ & 168.2 & Cibicidoides lamontdohertyi & 1.89 & 0.73 & 162 \\
\hline $19 \mathrm{H}-1,25-30$ & 168.2 & Cibicidoides kullenbergi & 1.74 & 0.83 & 162 \\
\hline $19 \mathrm{H}-1,75-80$ & 168.7 & $\begin{array}{l}\text { Cibicidoides kullenbergi } \\
\quad+\text { C. lamontdohertyi }\end{array}$ & 1.82 & 0.57 & 151 \\
\hline $19 \mathrm{H}-1,125-130$ & 169.2 & Cibicidoides kullenbergi & 1.85 & 0.61 & 88 \\
\hline $19 \mathrm{H}-2,25-30$ & 169.7 & Cibicidoides kullenbergi & 1.76 & 0.77 & 32 \\
\hline $19 \mathrm{H}-2,25-30$ & 169.7 & Cibicidoides lamontdohertyi & 1.65 & 0.71 & 32 \\
\hline $19 \mathrm{H}-2,70-75$ & 170.2 & Cibicidoides lamontdohertyi & 1.76 & 0.57 & 352 \\
\hline
\end{tabular}


Table 1 (continued).

\begin{tabular}{|c|c|c|c|c|c|}
\hline $\begin{array}{l}\text { Core, section, } \\
\text { interval }(\mathrm{cm})\end{array}$ & $\begin{array}{l}\text { Depth } \\
\text { (mbsf) }\end{array}$ & Species & $\delta^{18} \mathrm{O}$ & $\delta^{13} \mathrm{C}$ & $\begin{array}{l}\mathrm{P}: \mathrm{B} \\
\text { ratio }\end{array}$ \\
\hline \multicolumn{6}{|l|}{ 115-709B- (Cont.) } \\
\hline $19 \mathrm{H}-2,70-75$ & 170.2 & Cibicidoides kullenbergi & 2.06 & 0.77 & 352 \\
\hline $19 \mathrm{H}-2,125-130$ & 170.7 & Cibicidoides kullenbergi & 1.83 & 0.89 & 98 \\
\hline $19 \mathrm{H}-2,125-130$ & 170.7 & Cibicidoides lamontdohertyi & 2.03 & 0.58 & 98 \\
\hline $19 \mathrm{H}-3,25-30$ & 171.2 & Cibicidoides sp. C & 1.73 & 0.76 & 64 \\
\hline $19 \mathrm{H}-3,25-30$ & 171.2 & Cibicidoides kullenbergi & 1.80 & 0.72 & 64 \\
\hline $19 \mathrm{H}-3,25-30$ & 171.2 & Cibicidoides lamontdohertyi & 1.86 & 0.82 & 64 \\
\hline $19 \mathrm{H}-3,75-80$ & 171.7 & Cibicidoides sp. S & 2.01 & 0.93 & 160 \\
\hline $19 \mathrm{H}-3,125-130$ & 172.2 & Cibicidoides kullenbergi & 2.10 & 0.89 & 189 \\
\hline $19 \mathrm{H}-4,25-30$ & 172.7 & Cibicidoides kullenbergi & 2.06 & 0.90 & 138 \\
\hline $19 \mathrm{H}-4,75-80$ & 173.2 & Cibicidoides kullenbergi & 1.85 & 0.65 & 122 \\
\hline $19 \mathrm{H}-4,125-130$ & 173.7 & Cibicidoides kullenbergi & 1.61 & 0.58 & 114 \\
\hline $19 \mathrm{H}-5,25-30$ & 174.2 & Oridorsalis umbonatus & 2.08 & -0.10 & 67 \\
\hline $19 \mathrm{H}-5,25-30$ & 174.2 & Cibicidoides kullenbergi & 1.90 & 0.98 & 67 \\
\hline $19 \mathrm{H}-5,75-80$ & 174.7 & Cibicidoides lamontdohertyi & 2.09 & 0.91 & 105 \\
\hline $19 \mathrm{H}-5,75-80$ & 174.7 & Cibicidoides kullenbergi & 2.07 & 0.90 & 105 \\
\hline $19 \mathrm{H}-5,125-130$ & 175.2 & Cibicidoides lamontdohertyi & 1.96 & 0.74 & 229 \\
\hline $19 \mathrm{H}-5,125-130$ & 175.2 & Cibicidoides kullenbergi & 1.87 & 0.68 & 229 \\
\hline $19 \mathrm{H}-6,25-30$ & 175.7 & Cibicidoides kullenbergi & 1.65 & 0.48 & 185 \\
\hline $19 \mathrm{H}-6,75-80$ & 176.2 & Cibicidoides kullenbergi & 1.55 & 0.57 & 108 \\
\hline $19 \mathrm{H}-6,125-130$ & 176.7 & Cibicidoides sp. S & 1.90 & 0.86 & 409 \\
\hline $19 \mathrm{H}-7,27-30$ & 177.2 & Cibicidoides kullenbergi & 1.69 & 0.66 & 372 \\
\hline $21 \mathrm{H}-1,25-30$ & 187.4 & Cibicidoides kullenbergi & 2.09 & 1.50 & 210 \\
\hline $21 \mathrm{H}-1,25-30$ & 187.4 & Cibicidoides lamontdohertyi & 2.03 & 1.19 & 210 \\
\hline $21 \mathrm{H}-1,75-80$ & 187.9 & Cibicidoides lamontdohertyi & 1.96 & 1.30 & 178 \\
\hline $21 \mathrm{H}-1,125-130$ & 188.4 & Cibicidoides kullenbergi & 1.88 & 1.34 & 124 \\
\hline $21 \mathrm{H}-2,25-30$ & 188.9 & Cibicidoides lamontdohertyi & 2.32 & 1.64 & 387 \\
\hline $21 \mathrm{H}-2,25-30$ & 188.9 & Cibicidoides kullenbergi & 1.99 & 1.47 & 387 \\
\hline $21 \mathrm{H}-2,75-80$ & 189.4 & Cibicidoides lamontdohertyi & 2.15 & 1.50 & 280 \\
\hline $21 \mathrm{H}-2,125-130$ & 189.9 & Planulina renzi & 1.71 & 1.21 & 432 \\
\hline $21 \mathrm{H}-3,25-30$ & 190.4 & $\begin{array}{l}\text { Cibicidoides kullenbergi } \\
\quad+\text { C. lamontdohertyi }+ \text { Planulina renzi }\end{array}$ & 1.93 & 1.57 & 822 \\
\hline $21 \mathrm{H}-3,75-80$ & 190.9 & Cibicidoides lamontdohertyi & 1.84 & 1.06 & 223 \\
\hline $21 \mathrm{H}-3,123-128$ & 191.4 & $\begin{array}{l}\text { Cibicidoides lamontdohertyi } \\
+ \text { Planulina renzi }\end{array}$ & 1.81 & 1.42 & 162 \\
\hline $21 \mathrm{H}-4,25-30$ & 191.9 & $\begin{array}{l}\text { Cibicidoides kullenbergi } \\
\quad+\text { C. lamontdohertyi }\end{array}$ & 1.73 & 1.30 & 185 \\
\hline $21 \mathrm{H}-4,75-80$ & 192.4 & Cibicidoides lamontdohertyi & 2.12 & 1.40 & 551 \\
\hline $21 \mathrm{H}-4,125-130$ & 192.9 & $\begin{array}{l}\text { Cibicidoides kullenbergi } \\
\quad+\text { Planulina renzi }\end{array}$ & 1.64 & 0.90 & 278 \\
\hline $21 \mathrm{H}-5,25-30$ & 193.4 & $\begin{array}{l}\text { Cibicidoides lamontdohertyi } \\
\quad+C . \text { kullenbergi }\end{array}$ & 2.01 & 1.30 & 273 \\
\hline $21 \mathrm{H}-5,25-30$ & 193.4 & Cibicidoides kullenbergi & 2.18 & 1.46 & 273 \\
\hline $21 \mathrm{H}-5,75-80$ & 193.9 & Cibicidoides kullenbergi & 1.88 & 1.35 & 440 \\
\hline $21 \mathrm{H}-6,25-30$ & 194.9 & Cibicidoides kullenbergi & 2.12 & 1.53 & 234 \\
\hline $21 \mathrm{H}-6,75-80$ & 195.4 & Cibicidoides kullenbergi & 2.00 & 1.12 & 305 \\
\hline $21 \mathrm{H}-7,15-20$ & 196.4 & $\begin{array}{l}\text { Cibicidoides lamontdohertyi } \\
\quad+\text { C. kullenbergi }\end{array}$ & 1.98 & 1.09 & 206 \\
\hline $22 \mathrm{H}-1,25-30$ & 197.0 & Cibicidoides kullenbergi & 1.74 & 1.05 & 231 \\
\hline $22 \mathrm{H}-1,75-80$ & 197.5 & Cibicidoides kullenbergi & 1.71 & 1.18 & 135 \\
\hline $22 \mathrm{H}-1,125-130$ & 198.0 & $\begin{array}{l}\text { Cibicidoides kullenbergi } \\
+ \text { Planulina renzi }\end{array}$ & 1.50 & 1.14 & 161 \\
\hline $22 \mathrm{H}-2,25-30$ & 198.5 & Cibicidoides kullenbergi & 1.66 & 0.89 & 264 \\
\hline $22 \mathrm{H}-2,75-80$ & 199.0 & Cibicidoides kullenbergi & 1.61 & 0.58 & 288 \\
\hline $22 \mathrm{H}-2,125-130$ & 199.5 & Cibicidoides kullenbergi & 1.66 & 0.62 & 147 \\
\hline $22 \mathrm{H}-3,75-80$ & 200.5 & Cibicidoides kullenbergi & 1.65 & 0.72 & 218 \\
\hline
\end{tabular}

which the corrosiveness of Miocene intermediate and deep water are low may be intervals in which the waters have decreased concentrations of $\mathrm{CO}_{2}$ formed by oxidation of organic matter. Conversely, periods of poorer carbonate preservation may have been ones in which the concentration of respiratory $\mathrm{CO}_{2}$ in deep and intermediate water was higher.

\section{CONCLUSIONS}

The benthic isotopic record of Miocene Cibicidoides from Site 709 provides a record of oceanographic conditions at that location throughout the epoch. As expected, the record qualitatively resembles those of other DSDP and ODP sites. The data are consistent with the scenario for the evolution of thermohaline circulation in the Miocene Indian Ocean proposed by Woodruff and Savin (1989). Further testing of that scenario, however, requires isotopic data from other sites as well as resolution of the reasons for variability in interspecific differences between $\delta^{18} \mathrm{O}$ and $\delta^{13} \mathrm{C}$ values of different benthic taxa, especially Cibicidoides and Oridorsalis, from the same sample.

Correlation between $\delta^{13} \mathrm{C}$ values of Cibicidoides and P:B ratios at Site 709 suggests that Miocene intervals of poorer carbonate preservation at Site 709 are also intervals of increased concentration of respiratory $\mathrm{CO}_{2}$ in intermediate and deep waters.

\section{ACKNOWLEDGMENTS}

We thank the staff of the Ocean Drilling Program for their cooperation in providing the samples necessary for this study. This work was supported by National Science Foundation Grant Nos. OCE-8800449 (F.W.) and OCE-8800472 (S.M.S.). 


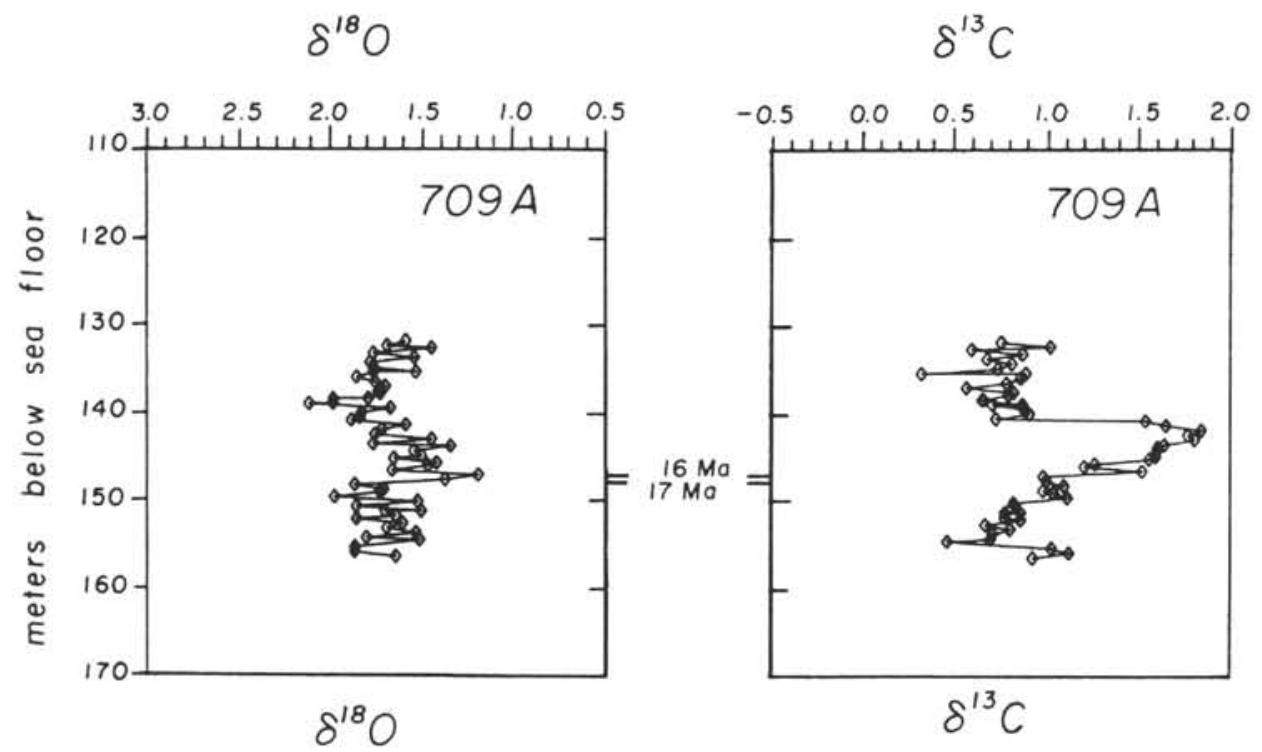

$\delta^{13} \mathrm{C}$

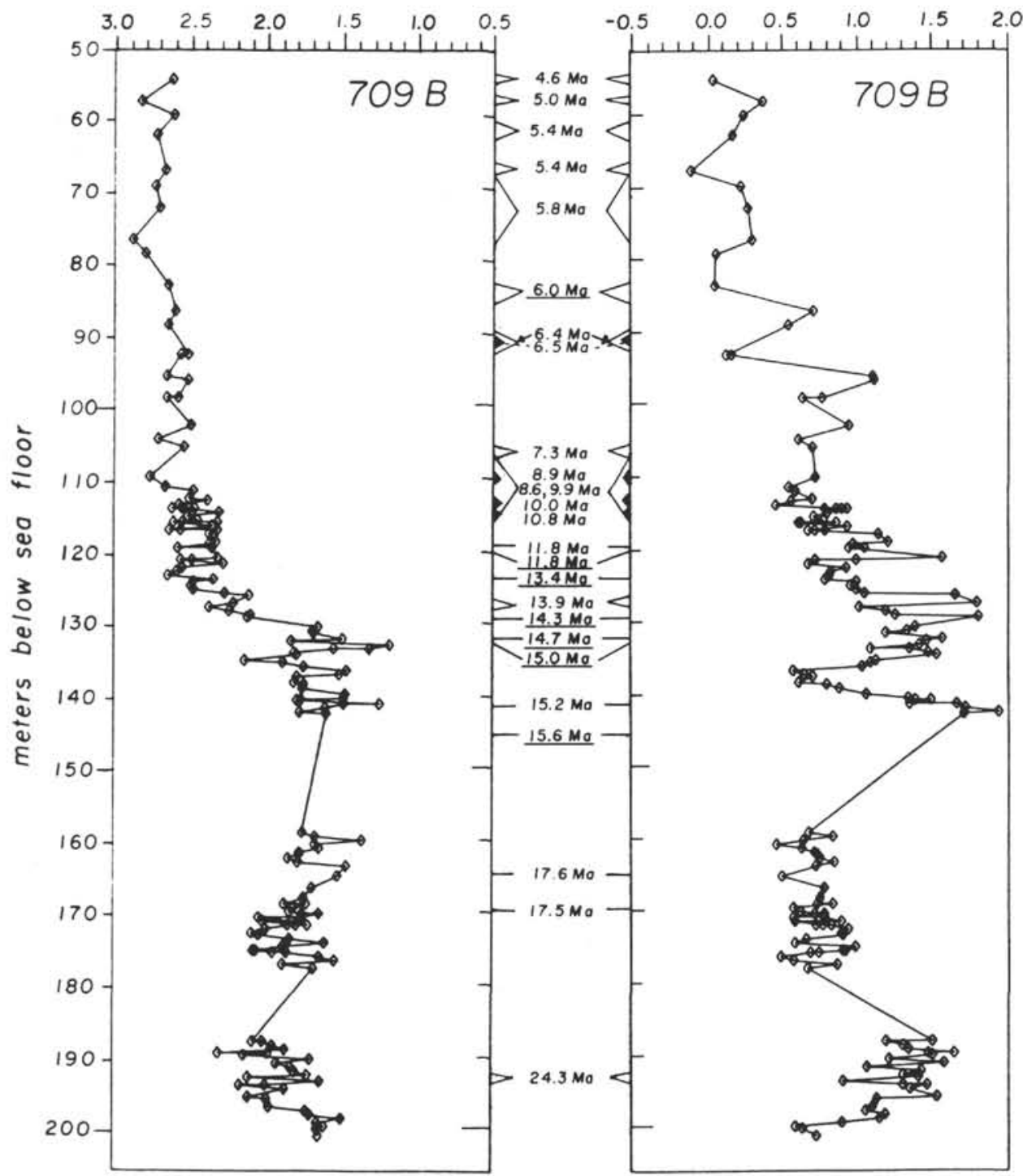

Figure 2. A. The $\delta^{18} \mathrm{O}$ and $\delta^{13} \mathrm{C}$ values of Cibicidoides from the Miocene section of Hole 709A. Inferred ages are shown. B. The $\delta^{18} \mathrm{O}$ and $\delta^{13} \mathrm{C}$ values of Cibicidoides from the Miocene section of Hole 709B. Isotopically derived ages (see text and Table 2 ) are underlined. Other ages are inferred from biostratigraphic data sources listed in Table 3. 
Table 2. Age determinations for Hole 709B based on the isotopic record (see text).

\begin{tabular}{cr}
\hline $\begin{array}{c}\text { Depth } \\
\text { (mbsf) }\end{array}$ & $\begin{array}{r}\text { Age } \\
(\mathrm{Ma})\end{array}$ \\
\hline $83-86$ & 6.0 \\
120 & 11.8 \\
124 & 13.4 \\
129 & 14.3 \\
132 & 14.7 \\
133 & 15.0 \\
146 & 15.6 \\
\hline
\end{tabular}

\section{REFERENCES}

Backman, J., Duncan, R. A., et al., 1988. Proc. ODP, Init. Repts., 115: College Station, TX (Ocean Drilling Program).

Barron, J., Keller, G., and Dunn, D. A., 1985. A multiple microfossil biochronology for the Miocene. In Kennett, J. P. (Ed.), The Miocene Ocean: Paleoceanography and Biogeography. Mem. Geol. Soc. Am., 163:21-36.

Duplessy, J.-C., Shackleton, N. J., Matthews, R. K., Prell, W. L., Ruddiman, W. F., Caralp, M., and Hendy, C. H., 1984. ${ }^{13} \mathrm{C}$ record of benthic foraminifera in the last interglacial ocean: implications for the carbon cycle and the global deep water circulation. Quat. Res., 21:225-243.

Epstein, S., Buchsbaum, S. R., Lowenstam, H. A., and Urey, H. C., 1951. Carbonate-water isotopic geothermometer. Geol. Soc. Am. Bull., 62:417-426.
Haq, B. U., Worsley, T. R., Burckle, L. H., Douglas, R. G., Keigwin, L. D., Opdyke, N. D., Savin., S. M., Sommer, M. A., Vincent, E., and Woodruff, F., 1980. Late Miocene carbon-isotopic shift and synchroneity of some phytoplanktonic biostratigraphic events. Geology, 8:425-431.

Miller, K. G., and Katz, M., 1987. Oligocene to Miocene benthic foraminiferal and abyssal circulation changes in the North Atlantic. Micropaleontology, 33:130.

Savin, S. M., and Woodruff, F., 1990. Isotopic evidence for temperature and productivity in the Tertiary oceans. In Burnett, W. C., and Riggs, S. R. (Eds.), Phosphate Deposits of the World: Genesis of Neogene to Recent Phosphorites (Vol. 3): Cambridge (Cambridge Univ. Press), 241-259.

Shackleton, N. J., Hall, M. A., and Boersma, A. J., 1984. Oxygen and carbon isotope data from Leg 74 foraminifers. In Moore, T. C., Jr., Rabinowitz, P. D., Init. Repts. DSDP, 74: Washington (U.S. Govt. Printing Office), 599-612.

Vincent, E., Killingley, J. S., and Berger, W. H., 1982. The magnetic epoch- 6 carbon shift: a change in the oceans $\mathrm{C}^{13} / \mathrm{C}^{12}$ ratio. Mar. Micropaleontol., 5:185-203.

1985. Miocene oxygen and carbon isotope stratigraphy of the tropical Indian Ocean. In Kennett, J. P. (Ed.), The Miocene Ocean: Paleoceanography and Biogeography. Mem. Geol. Soc. Am., 163: 103-130.

Woodruff, F., and Savin, S. M., 1989. Miocene deep-water oceanography. Paleoceanography, 4:87-140.

Date of initial receipt: 15 June 1989

Date of acceptance: 20 December 1989

Ms 115B-147

Table 3. Hole 709B Biostratigraphic age datums from Backman, Duncan, et al. (1988), Rio et al. (this volume), and Johnson (this volume) that are consistent with the isotopic data.

\begin{tabular}{|c|c|c|c|}
\hline $\begin{array}{l}\text { Depth } \\
\text { (mbsf) }\end{array}$ & $\begin{array}{l}\text { Age } \\
\text { (Ma) }\end{array}$ & Datum & Zonal boundary \\
\hline $53.3-54.8$ & 4.6 & Ceratolithus rugosus $(\mathrm{N})(\mathrm{B})$ & $\mathrm{CN} 10 \mathrm{c} / \mathrm{CN} 10 \mathrm{~b}, \mathrm{NN} 13 / \mathrm{NN} 12$ \\
\hline $56.3-57.8$ & 5.0 & Ceratolithus acutus $(\mathrm{N})(\mathrm{B})$ & $\mathrm{CN} 10 \mathrm{~b} / \mathrm{CN} 10 \mathrm{a}$ \\
\hline $60.8-63.0$ & 5.4 & Discoaster quinqueramus $(\mathrm{N})(\mathrm{T})$ & $\mathrm{CN} 10 \mathrm{a} / \mathrm{CN} 9$ \\
\hline $66.5-68.0$ & 5.4 & Acrobotrys tritubus (R) & \\
\hline $68.0-77.6$ & 5.8 & Pulleniatina primalis $(\mathrm{F})(\mathrm{B})$ & $\mathrm{N} 17 \mathrm{~b} / \mathrm{N} 17 \mathrm{a}$ \\
\hline $89.5-92.5$ & 6.4 & Stichocorys delmontensis to $S$. peregrina $(\mathrm{R})$ & \\
\hline $90.2-91.9$ & 6.5 & Amaurolithus primus $(\mathrm{N})(\mathrm{B})$ & $\mathrm{CN} 9 \mathrm{~b} / \mathrm{CN} 9 \mathrm{a}$ \\
\hline $105.8-107.3$ & 7.3 & Discoaster quinqueramus (N)(B) & $\mathrm{NN} 11 / \mathrm{NN} 10$ \\
\hline $107-116$ & 8.6 & Neogloquadrina acostaensis (F)(B) & N16/N15 \\
\hline $107-116$ & 9.9 & Globorotalia siakensis $(\mathrm{F})(\mathrm{T})$ & $\mathrm{N} 15 / \mathrm{N} 14$ \\
\hline $109.1-111.1$ & 8.9 & Discoaster hamatus $(\mathrm{N})(\mathrm{T})$ & CN8a/CN7, NN10/NN9 \\
\hline $112.7-114.2$ & 10.0 & Discoaster hamatus (N)(B) & $\mathrm{CN} 7 \mathrm{a} / \mathrm{CN} 6, \mathrm{NN} 9 / \mathrm{NN} 8$ \\
\hline $114.2-115.7$ & 10.8 & Catinaster coalitus $(\mathrm{N})(\mathrm{B})$ & CN6/CN5, NN8/NN7 \\
\hline 119 & 11.8 & Sphaeroidinella subdehiscens $(\mathrm{F})(\mathrm{T})$ & $\mathrm{N} 13 / \mathrm{N} 12$ \\
\hline $126.5-127.7$ & 13.9 & Sphenolithus heteromorphus (N)(T) & CN5A/CN4, NN6/NN5 \\
\hline 141 & 15.2 & Orbulina suturalis $(\mathrm{F})(\mathrm{B})$ & $\mathrm{N} 9 / \mathrm{N} 8$ \\
\hline 164.6 & 17.6 & Catapsydrax dissimilis $(\mathrm{F})(\mathrm{T})$ & N7/N6 \\
\hline $169.8-169.9$ & 17.5 & Sphenolithus heteromorphus $(\mathrm{N})(\mathrm{B})$ & $\mathrm{CN} 3 / \mathrm{CN}_{2}$ \\
\hline $192.2-193.5$ & 24.3 & Lychnocanoma elongata $(\mathrm{R})(\mathrm{B})$ & Zonal base \\
\hline
\end{tabular}

Notes: $\mathrm{F}=$ foraminifer, $\mathrm{R}=$ radiolarian, $\mathrm{N}=$ nannoplankton, $\mathrm{T}=\operatorname{range}$ top, and $\mathrm{B}=$ range bottom. 


\section{$\delta$ values}

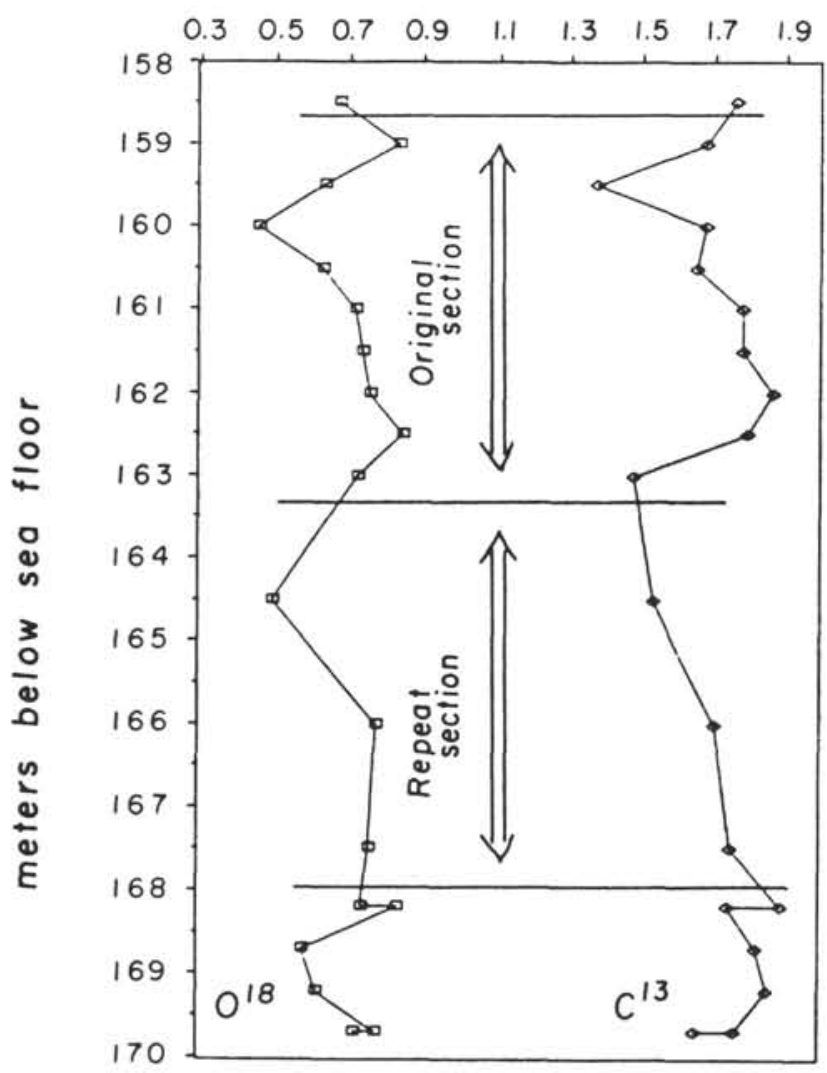

Figure 3. Detail of the oxygen and carbon isotopic records of Hole 709B Cibicidoides over the interval from 155 to 170 mbsf. Backman, Duncan, et al. (1988) inferred from whole-sample magnetic susceptibility data that the section between 163.3 and 168 mbsf was a repeat of the immediately overlying interval. The isotopic data are consistent with, but do not require, that interpretation. Squares $=\delta^{18} \mathrm{O}$ values, and diamonds indicate $\delta^{13} \mathrm{C}$ values.

Table 4. Mean $\delta^{18} \mathrm{O}$ and $\delta^{13} \mathrm{C}$ values of Cibicidoides from Miocene time intervals, Site 709.

\begin{tabular}{|c|c|c|c|c|c|c|}
\hline \multirow{2}{*}{$\begin{array}{c}\text { Interval } \\
(\mathrm{Ma})\end{array}$} & \multicolumn{3}{|c|}{$\delta^{18} \mathrm{O}$} & \multicolumn{3}{|c|}{$\delta^{13} \mathrm{C}$} \\
\hline & Average & SD & $N$ & Average & SD & $N$ \\
\hline $5-6$ & 2.74 & 0.09 & 9 & 0.17 & 0.15 & 9 \\
\hline $6-7$ & 2.60 & 0.06 & 10 & 0.61 & 0.38 & 10 \\
\hline $7-10$ & 2.59 & 0.13 & 7 & 0.63 & 0.07 & 7 \\
\hline $10-12$ & 2.48 & 0.11 & 20 & 0.83 & 0.18 & 20 \\
\hline $12-14$ & 2.41 & 0.15 & 16 & 1.06 & 0.32 & 16 \\
\hline $14-15$ & 1.68 & 0.30 & 9 & 1.38 & 0.20 & 9 \\
\hline $15-16$ & 1.69 & 0.18 & 53 & 1.10 & 0.42 & 53 \\
\hline $16-17$ & 1.41 & 0.19 & 3 & 1.16 & 0.25 & 3 \\
\hline $17-18$ & 1.72 & 0.13 & 36 & 0.77 & 0.16 & 36 \\
\hline $18-20$ & 1.88 & 0.16 & 21 & 0.75 & 0.14 & 21 \\
\hline $21-23$ & 1.99 & 0.08 & 4 & 1.33 & 0.11 & 4 \\
\hline $23-24$ & 1.95 & 0.21 & 9 & 1.36 & 0.23 & 9 \\
\hline
\end{tabular}

Note: $\mathrm{SD}=$ standard deviation and $N=$ number of comparisons.
Table 5. Intra-sample comparisons of $\delta^{18} \mathrm{O}$ values of Miocene benthic foraminifers, Site 709.

\begin{tabular}{lcccc}
\hline & $\begin{array}{c}\text { Cibicidoides } \\
\text { kullenbergi }\end{array}$ & $\begin{array}{c}\text { Cibicidoides } \\
\text { whellerstorfi }\end{array}$ & $\begin{array}{c}\text { Cibicidoides } \\
\text { lamontdohertyi }\end{array}$ & $\begin{array}{c}\text { Oridorsalis } \\
\text { umbonatus }\end{array}$ \\
\hline $\begin{array}{l}\text { Cibicidoides } \\
\text { kullenbergi }\end{array}$ & - & $-0.11 \pm 0.14$ & $+0.03 \pm 0.17$ & $+0.34 \pm 0.11$ \\
$\begin{array}{l}\text { Cibicidoides } \\
\text { wuellerstorfi }\end{array}$ & $-4=4$ & $N=10$ & $N=5$ \\
$\begin{array}{l}\text { Cibicidoides } \\
\text { lamontdohertyi }\end{array}$ & - & $+0.19 \pm 0.08$ & $+0.47 \pm 0.03$ \\
$\begin{array}{l}\text { Oridorsalis } \\
\text { umbonatus }\end{array}$ & & & - & No comparison \\
\hline
\end{tabular}

Note: Differences are calculated as the $\delta^{18} \mathrm{O}$ value of species in column minus species in row and are listed as averages \pm 1 SD. For example, the average of $\delta^{18} \mathrm{O}_{\text {wuellerstorf } i}$ minus $\delta^{18} \mathrm{O}_{\text {kullenbergi }}$ is $+0.11 \pm 0.14 . N=$ number of comparisons.

Table 6. Intra-sample comparisons of $\delta^{13} \mathrm{C}$ values of Miocene benthic foraminifers, Site 709.

\begin{tabular}{|c|c|c|c|c|}
\hline & $\begin{array}{c}\text { Cibicidoides } \\
\text { kullenbergi }\end{array}$ & $\begin{array}{l}\text { Cibicidoides } \\
\text { wuellerstorfi }\end{array}$ & $\begin{array}{c}\text { Cibicidoides } \\
\text { lamontdohertyi }\end{array}$ & $\begin{array}{l}\text { Oridorsalis } \\
\text { umbonatus }\end{array}$ \\
\hline $\begin{array}{l}\text { Cibicidoides } \\
\text { kullenbergi }\end{array}$ & - & $\begin{array}{l}-0.07 \pm 0.08 \\
N=4\end{array}$ & $\begin{array}{l}-0.10 \pm 0.14 \\
N=12\end{array}$ & $\begin{array}{l}+0.90 \\
N=5\end{array}$ \\
\hline $\begin{array}{l}\text { Cibicidoides } \\
\text { wuellerstorfi }\end{array}$ & & - & $\begin{array}{l}-0.22 \\
N=3\end{array}$ & $\begin{array}{l}-1.20 \pm 0.02 \\
N=2\end{array}$ \\
\hline $\begin{array}{l}\text { Cibicidoides } \\
\text { lamontdohertyi }\end{array}$ & & & - & No comparison \\
\hline $\begin{array}{l}\text { Oridorsalis } \\
\text { umbonatus }\end{array}$ & & & & - \\
\hline
\end{tabular}

Note: Differences are listed as averages $\pm 1 \mathrm{SD} . N=$ number of comparisons. 


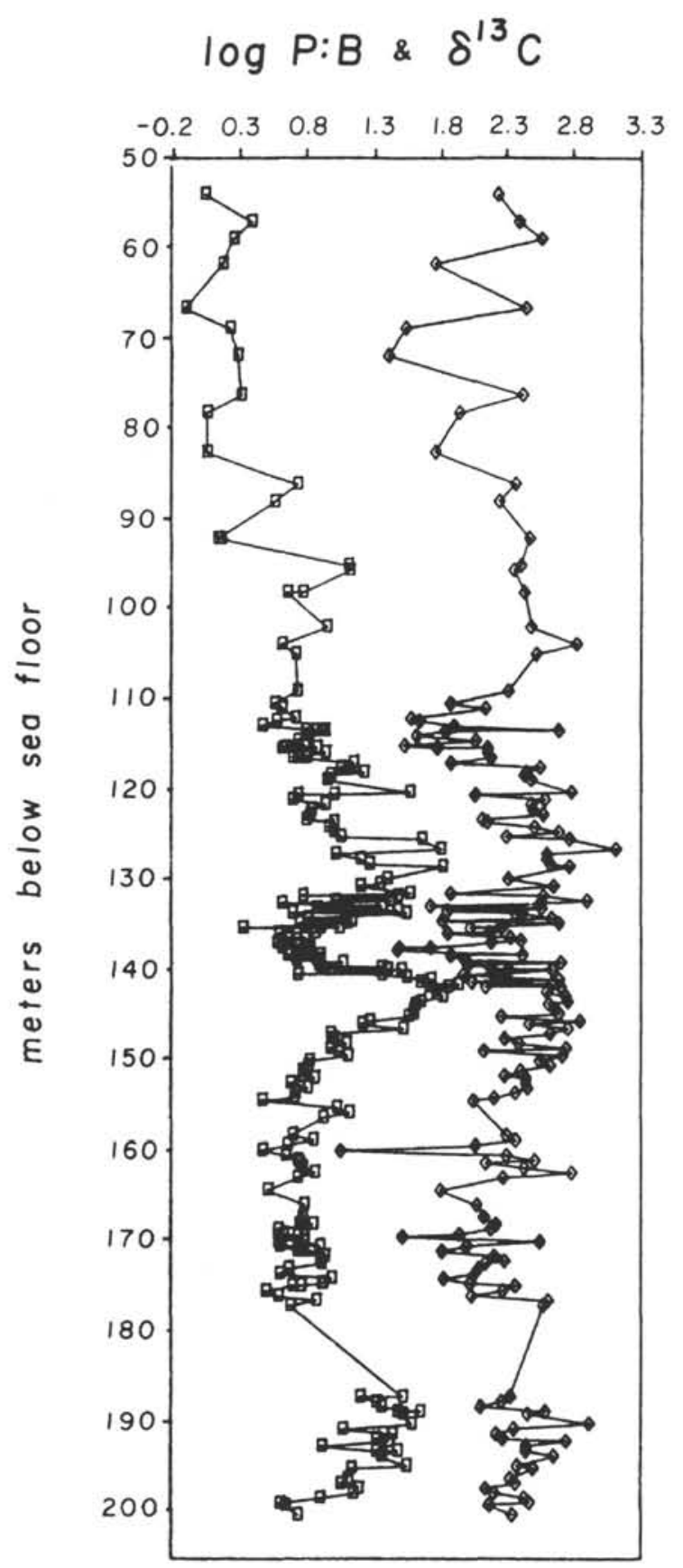

Figure 4. Time-series plot of $\delta^{13} \mathrm{C}$ values of Cibicidoides from Holes 709A and 709B (squares) compared with planktonic:benthic ratios of the sediment samples (diamonds). P:B ratios are plotted on a logarithmic scale. Major increases in $\delta^{13} \mathrm{C}$ correspond to increases in P:B ratio.

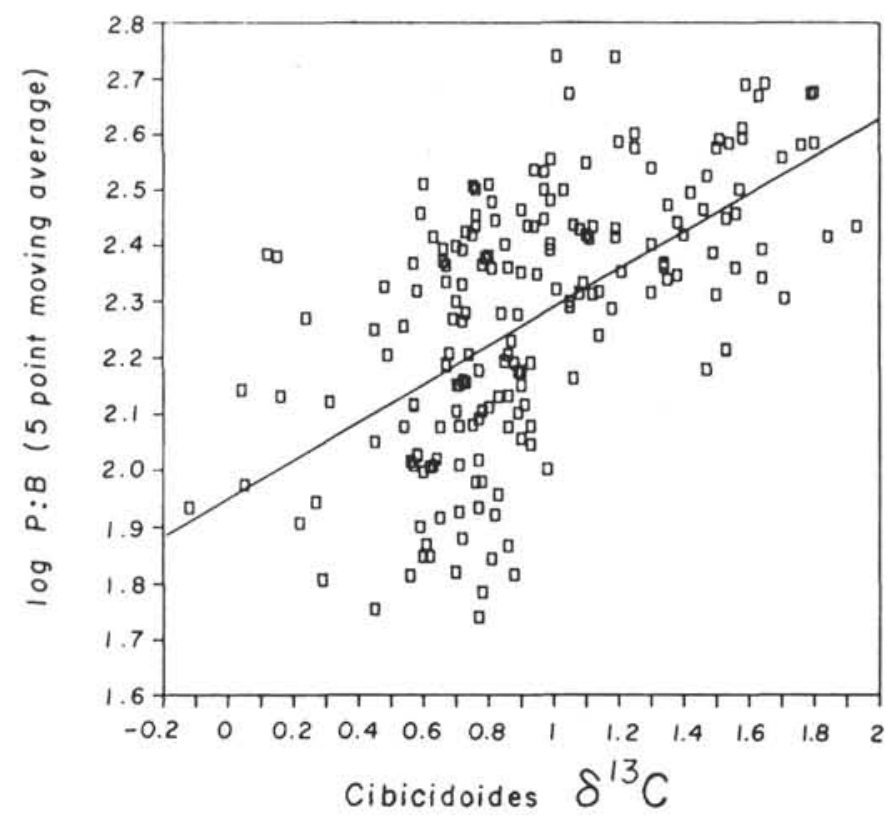

Figure 5. Scatter plot of $\delta^{13} \mathrm{C}$ values of Cibicidoides vs. the logarithm of the $\mathrm{P}: \mathrm{B}$ ratio (5-point moving average). The regression line is significant at the $99 \%$ level. 